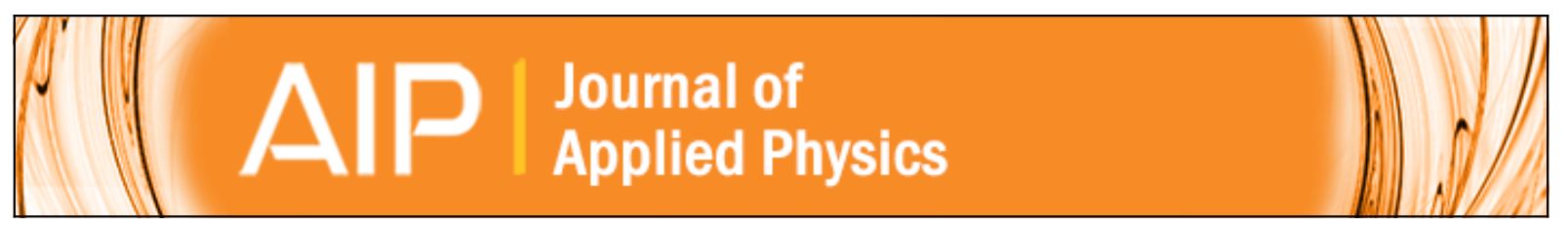

Poole-Frenkel effect and variable-range hopping conduction in metal/YBCO resistive switching devices
A. Schulman, L. F. Lanosa, and C. Acha

Citation: Journal of Applied Physics 118, 044511 (2015); doi: 10.1063/1.4927522

View online: http://dx.doi.org/10.1063/1.4927522

View Table of Contents: http://scitation.aip.org/content/aip/journal/jap/118/4?ver=pdfcov

Published by the AIP Publishing

Articles you may be interested in

Meyer-Neldel rule and Poole-Frenkel effect in chalcogenide glasses

J. Appl. Phys. 114, 023707 (2013); 10.1063/1.4813128

Temperature dependence of the resistance switching effect studied on the metal/ YBa $2 \mathrm{Cu} 3 \mathrm{O} 6+\mathrm{x}$ planar junctions

J. Vac. Sci. Technol. B 29, 01 AD04 (2011); 10.1116/1.3521408

Sensitivity of nanotube chemical sensors at the onset of Poole-Frenkel conduction

Appl. Phys. Lett. 96, 163110 (2010); 10.1063/1.3379291

Analysis of hopping conduction in semiconducting and metallic carbon nanotube devices

J. Appl. Phys. 105, 124309 (2009); 10.1063/1.3151916

High-power-density fault-current limiting devices using superconducting YBa $2 \mathrm{Cu} 3 \mathrm{O} 7$ films and high-resistivity alloy shunt layers

Appl. Phys. Lett. 85, 4427 (2004); 10.1063/1.1812572

Did your publisher get

18 MILLION DOWNLOADS in 2014?

AIP Publishing did.

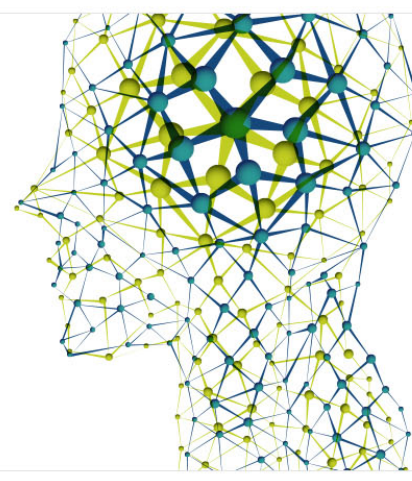




\title{
Poole-Frenkel effect and variable-range hopping conduction in metal/YBCO resistive switching devices
}

\author{
A. Schulman, L. F. Lanosa, and C. Acha ${ }^{\text {a) }}$ \\ Laboratorio de Bajas Temperaturas, Departamento de Física, FCEyN - Universidad de Buenos Aires \\ and IFIBA - CONICET, Pabellón I, Ciudad Universitaria, C1428EHA Buenos Aires, Argentina
}

(Received 2 March 2015; accepted 16 July 2015; published online 31 July 2015)

\begin{abstract}
Current-voltage (IV) characteristics and the temperature dependence of the contact resistance $[R(T)]$ of $\mathrm{Au} / \mathrm{YBa}_{2} \mathrm{Cu}_{3} \mathrm{O}_{7-\delta}$ (optimally doped YBCO) interfaces have been studied at different resistance states. These states were produced by resistive switching after accumulating cyclic electrical pulses of increasing number and voltage amplitude. The IV characteristics and the $R(T)$ dependence of the different states are consistent with a Poole-Frenkel (P-F) emission mechanism with trapping-energy levels $E_{t}$ in the $0.06-0.11 \mathrm{eV}$ range. $E_{t}$ remains constant up to a number-of-pulses-dependent critical voltage and increases linearly with a further increase in the voltage amplitude of the pulses. The observation of a P-F mechanism reveals the existence of an oxygen-depleted layer of YBCO near the interface. A simple electrical transport scenario is discussed, where the degree of disorder, the trap energy level, and the temperature range determine an electrical conduction dominated by non-linear effects, either in a P-F emission or in a variable-range hopping regime. (C) 2015 AIP Publishing LLC. [http://dx.doi.org/10.1063/1.4927522]
\end{abstract}

\section{INTRODUCTION}

There is a technological need to develop faster, smaller, cheaper, and more reliable memory devices, which is therefore promoting the search to improve their capacity to retain a higher density of information, at lower costs, using less energy and operating under hostile environment conditions. ${ }^{1,2}$ As possible candidates to fulfill such an overwhelming task, we can find memories (RRAM) based on the resistive switching (RS) mechanism, where the non-volatile and reversible resistance state of a metal-oxide interface is varied upon the application of electric pulses. ${ }^{3}$ In recent years, some remarkable improvements have been made to understand the physics behind the memory properties of RRAM devices. ${ }^{4}$ As an example, the underlying memory mechanism of devices based on metal-perovskite oxide junctions was associated with the resistance change due to oxygen-vacancy electromigration near the interface. ${ }^{5}$ Despite these advances, many aspects are still unclear and should be addressed in order to improve their practical capabilities. In that sense, one important feature to determine in each particular device is the conduction mechanism that dominates its electrical transport properties. This knowledge can be the key to control most of the desired properties of a memory device as it points out which are the relevant microscopic factors that determine their resistive state. Depending on material characteristics of the metal-oxide interface, the mechanism can be electrode-limited or bulk-limited. ${ }^{6,7}$ In the electrode-limited ones, the work function of the metal, the carrier affinity, and the thickness of the oxide determine the barrier height and the probability to produce an electricfield-induced-current through the junction. In this case, the mechanism can be described as Schottky, Fowler-Nordheim,

\footnotetext{
a) Author to whom correspondence should be addressed. Electronic mail: acha@df.uba.ar
}

or direct tunneling emission. In the bulk-limited case, the conduction mechanism is determined by the electrical properties of the oxide near the interface, particularly by the existence of traps and their energy levels. Poole-Frenkel (P-F) emission and space-charge-limited conduction (SCLC) are two examples of transport mechanisms influenced by the energy distribution and density of traps.

Metal- $\mathrm{YBa}_{2} \mathrm{Cu}_{3} \mathrm{O}_{7-\delta}$ (YBCO) interfaces on ceramic and thin-films samples have both shown bipolar RS characteristics. $^{8-11}$ Their retentivity ${ }^{12,13}$ as well as their response to cyclic electric field stresses ${ }^{14}$ have been previously studied. Although the microscopic origin of its RS properties was successfully associated with the electromigration of oxygen vacancies, ${ }^{5}$ no detailed studies of the conduction mechanism through the interface have been already performed.

In this paper, our goal is to identify the relevant transport mechanism of $\mathrm{Au} / \mathrm{YBa}_{2} \mathrm{Cu}_{3} \mathrm{O}_{7-\delta}(\mathrm{YBCO})$ interfaces, in order to point out the microscopic factors that determine the resistive state, as the temperature dependence and magnitude of the resistance change upon producing a RS. Our results indicate that a P-F emission mechanism dominates the current-voltage dependence of the junction, consequently indicating the existence of carrier traps and of a low conductivity region in the interfacial YBCO. Within this scenario, most probably related to a random distribution of oxygen vacancies near the junction, we present a description of the electrical conduction based on a modified Variable-Range Hopping (VRH) mechanism. The modification that we propose is based on considering that the available electrical carriers are only those thermally or voltage assisted de-trapped which obey a P-F law. In this framework, we show that increasing the amplitude or the number of applied pulses has qualitatively the same microscopic effect on modifying the trap energy level as well as the resistivity and the geometric factor of a conducting channel. 


\section{EXPERIMENTAL DETAILS}

The device was prepared by sputtering four metallic electrodes on top of a face of a good quality and optimally doped YBCO textured ceramic slab $\left(8 \times 4 \times 0.5 \mathrm{~mm}^{3}\right.$, see the inset of Fig. 1). The YBCO slab was synthesized following a similar procedure to the one described in Ref. 15 The homogeneity and the oxygen content were checked by measuring its resistive superconducting transition, which showed a $10 \%$ $90 \%$ width of $\simeq 2 \mathrm{~K}$ and a $T_{c}^{\text {onset }}=(90 \pm 0.2) \mathrm{K}$ (see Ref. 8). By considering the relation between $T_{c}$ and the mean oxygen content $(7-\delta),{ }^{16,17}$ we estimated that $\delta \leqq 0.05$. The sputtered electrodes have a $1 \times 1 \mathrm{~mm}^{2}$ area and a mean separation of $0.5 \mathrm{~mm}$. Copper leads were carefully fixed by using silver paint, without contacting directly the surface of the sample. $\mathrm{Au}$ and $\mathrm{Pt}$ were chosen as metals for the pair of pulsed electrodes, labeled 1 and 2, respectively. As we have previously shown, ${ }^{18}$ the $\mathrm{Pt} / \mathrm{YBCO}$ interfaces have a lower resistance value than the $\mathrm{Au} / \mathrm{YBCO}$ ones $\left(R_{P t} \leqslant R_{A u} / 3\right)$ and a small RS amplitude. Thus, we may disregard the influence of the $\mathrm{Pt} /$ YBCO electrode and proceed as if only the $\mathrm{Au} / \mathrm{YBCO}$ electrode were active (i.e., presents a relevant RS effect), simplifying the effects produced upon voltage pulsing treatments.

In order to analyze the sensitivity of the microscopic parameters that control the electrical transport properties of the $\mathrm{Au} / \mathrm{YBCO}$ interface to the voltage amplitude and to the number of applied pulses, we applied the following pulsing protocol (see Fig. 1).

Temperature is initially stabilized at $300 \mathrm{~K}$, with the active $\mathrm{Au} / \mathrm{YBCO}$ electrode set to its low resistance state (LRS) $\left(R_{A u}=R^{L R S}\right)$. A Pt thermometer is used to determine the temperature of the device. To produce a RS to a high resistance state (HRS) $\left(R_{A u}=R^{H R S}\right)$, a "reset" burst of $N$ positive unipolar pulses $(500 \mu$ s width at $1 \mathrm{kHz}$ rate $)$ of a $V_{\text {pulse }}$ amplitude $\left(2 \mathrm{~V} \leq V_{\text {pulse }} \leq 5 \mathrm{~V}\right)$ is applied with an Agilent 33250A waveform generator to electrodes 1 and 2, during a time $t_{0}$ (from 10 s to $500 \mathrm{~s}$, depending on the $N$ value). We want to note here that the polarity of the pulses was defined arbitrarily with the

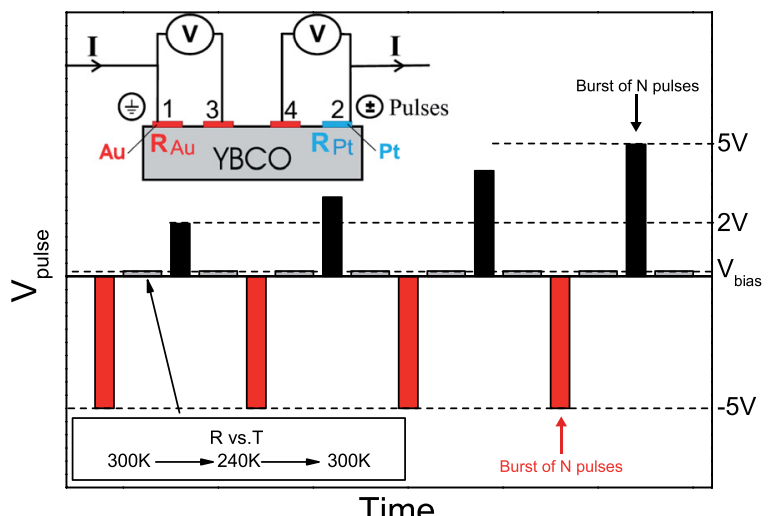

FIG. 1. Diagram of the pulsing protocol used to study the effect of the amplitude and of the number of pulses on the RS of the Au/YBCO interface. The black bars correspond to the reset process, while the red ones to the set. Each bar represents a burst of $\mathrm{N}$ pulses. The grey areas correspond the $R(T)$ measurement with a small bias current; the protocol was repeated for each number of pulses per burst $(\mathrm{N})$. The inset shows the YBCO ceramic device and its electrode configuration. Au was sputtered on pads 1, 3, and 4 while Pt on pad 2. See the text for the details of the measuring procedure. ground terminal connected to the active $\mathrm{Au} / \mathrm{YBCO}$ contact. With this definition, for example, a negative pulse produces a positive voltage drop on the $\mathrm{Au} / \mathrm{YBCO}$ interface. In order to avoid overheating effects on the resistance measurements, a time equal to $t_{0}$ is waited before measuring the temperature dependence of remnant resistance $R^{H R S}(T)$. Then, this resistance is measured as a function of temperature, cooling down to $240 \mathrm{~K}$ and heating back to $300 \mathrm{~K}$ at a $2 \mathrm{~K}$ per minute rate. For that, a DC three terminal method is used, by applying a small bias current $(100 \mu \mathrm{A})$ to electrodes 1 and 2 and by measuring the voltage difference $(\Delta V<0.1 \mathrm{~V})$ between electrodes 1-3. A Keithley 224 was used as the current source and an Agilent $34420 \mathrm{~A}$ as the nanovoltmeter. Voltage was also measured between electrodes 2-4 to check the small RS effect of the $\mathrm{Pt} / \mathrm{YBCO}$ electrode.

Also using this configuration, DC current-voltage characteristics (IV) were measured at $300 \mathrm{~K}$, where a small I-V range was explored in order to avoid an RS of the active electrode. Corrections to $R^{H R S}$ by considering the resistance of the bulk YBCO (beyond the interfacial zone) are negligible taking into account that its value is only $\simeq 0.1 \Omega \ll 50 \Omega<$ $R_{A u}$ in this temperature range.

After the $R^{H R S}(T)$ measurement, due to the bipolar nature of the RS in the metal/perovskite interface, a "set" burst of maximum opposite polarity $\left(-V_{\text {pulse }}^{\max }=-5 V\right)$ of the same number of pulses of the "reset" burst is applied to come back to a LRS and to erase the cumulative effects of the reset pulses. Then, the temperature dependence of the LRS remnant resistance is measured $\left[R^{L R S}(T)\right]$ following the same procedure as with $R^{H R S}(T)$. The process is then completely repeated for a new $V_{\text {pulse }}$ value, increased with a fixed step, until it reaches our experimental maximum $\left(V_{\text {pulse }}^{\max }=5 \mathrm{~V}\right)$. The whole process was performed for different numbers of pulses $N$ in the $10^{4} \leq N \leq 5 \times 10^{5}$ range.

\section{RESULTS AND DISCUSSION}

In Fig. 2(a), the hysteretical remnant resistance of both $\mathrm{Au} / \mathrm{YBCO}$ and $\mathrm{Pt} / \mathrm{YBCO}$ contacts $\left(R_{A u}\right.$ and $R_{P t}$, respectively) after applying a burst of $100 \times 10^{3}$ pulses of amplitude $V_{\text {pulse }}$ can be observed. As $V_{\text {pulse }}$ varied following a loop, these curves are usually called Resistance Hysteresis Switching Loop (RHSL). The observed bipolar memory behavior of both contacts and their complementary response to $V_{\text {pulse }}$ is a typical characteristic of the RRAM devices. ${ }^{5,9,11}$ The lower switching amplitude and the better stability of $R_{P t}$ in comparison to $R_{A u}$, in accordance with the expected passivity of the former can be noticed. Taking this into account, hereafter our results and discussions will be only related to the $\mathrm{Au} / \mathrm{YBCO}$ active electrode.

In Fig. 2(b), the relative amplitude of the remnant resistance change at room temperature, defined as $\alpha=\left(R^{H R S}\right.$ $\left.-R^{L R S}\right) / R^{L R S}$, is presented as a function of $V_{\text {pulse }}$ and for different numbers of applied pulses $(N)$. As shown in a previous study, ${ }^{18} \alpha$ increases logarithmically with increasing $N$ and follows a power-law-like dependence with $V_{\text {pulse }}$ (if $V_{\text {pulse }}$ is higher than an $N$-dependent threshold voltage, $V_{c}(N)$ ). A similar result has been observed for $\mathrm{Ag} /$ manganite interfaces and was interpreted as a consequence of the voltage and history dependent spatial distribution of oxygen-vacancies near the interface. ${ }^{19}$ 

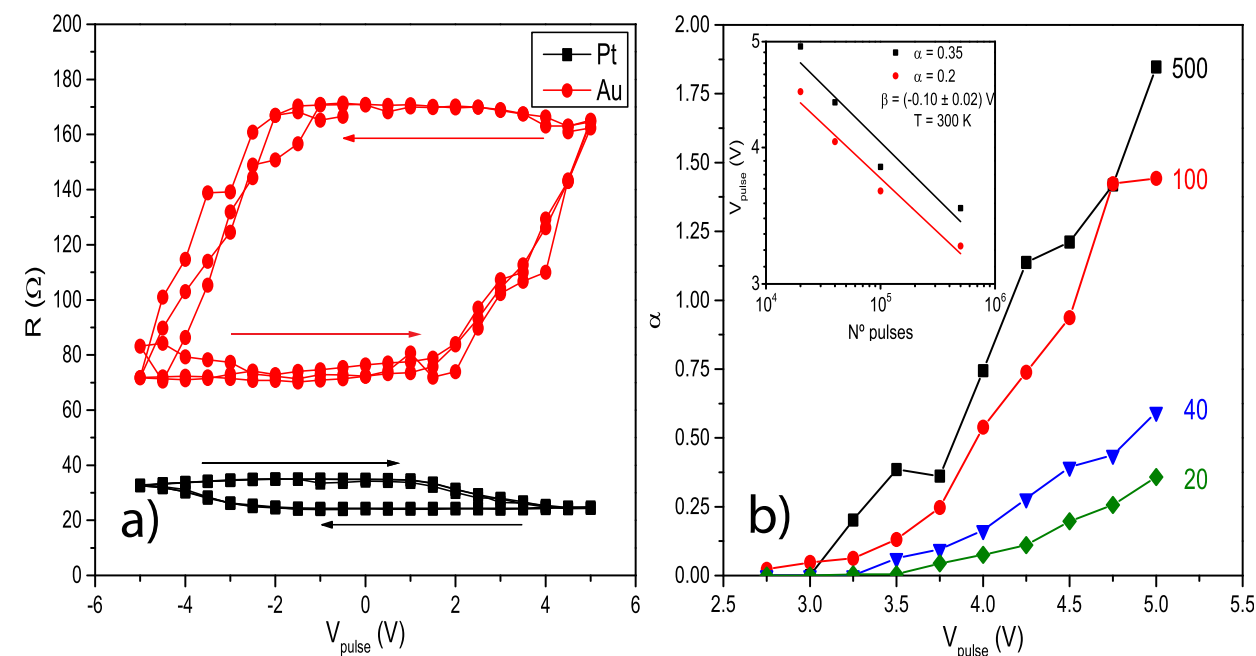

FIG. 2. (a) RHSL for both pulsed contacts $\left(\mathrm{N}=100 \times 10^{3}\right)$. The $\mathrm{Pt} / \mathrm{YBCO}$ interface shows lower resistance and smaller RS amplitude than Au/YBCO. (b) Relative variation of the remnant resistance $\left[\alpha=\left(R^{H R S}-R^{L R S}\right) / R^{L R S}\right]$ of the $\mathrm{Au} / \mathrm{YBCO}$ interface as a function of the amplitude of the reset pulses $\left(V_{\text {pulse }}\right)$ with a different number of square pulses per burst: $N=20 \times 10^{3}$, $40 \times 10^{3}, 100 \times 10^{3}$, and $500 \times 10^{3}$. The inset shows the analog to the Basquin curves ( $V-N$ curves) at $300 \mathrm{~K}$ for two arbitrary failure criteria $\alpha=0.2$ and 0.35 (see Ref. 18).
The inset of Fig. 2 shows the $V_{\text {pulse }}-\mathrm{N}$ curves that produce different $\alpha$ values ( 0.2 and 0.35 ), which recalls a similar behavior to that described by the Basquin law of mechanical stress-lifetime test, which corresponds to ${ }^{20,21} V_{p} \sim N^{\beta}$, with $\beta \simeq-0.1$.

From the IV characteristics obtained for both states at $300 \mathrm{~K}$, we have plotted a typical result as $\ln (V / I)$ vs $V^{1 / 2}$ in Fig. 3. Data are well fitted by a linear dependence, although there are some small deviations at low voltages. This result indicates that the bulk-limited P-F emission is the dominant mechanism for the electrical transport of carriers through this interface. This can be seen by considering the expression of the P-F current $\left(I_{P-F}\right)$ as a function of the voltage $V$ at a fixed temperature $T$, which corresponds to ${ }^{22}$

$$
I_{P-F}=\left(R_{0}\right)^{-1} V \exp \left[-E_{t} /\left(k_{B} T\right)+B V^{1 / 2}\right],
$$

with

$$
B=\frac{q^{3 / 2}}{k_{B} T(\pi \epsilon d)^{1 / 2}},
$$

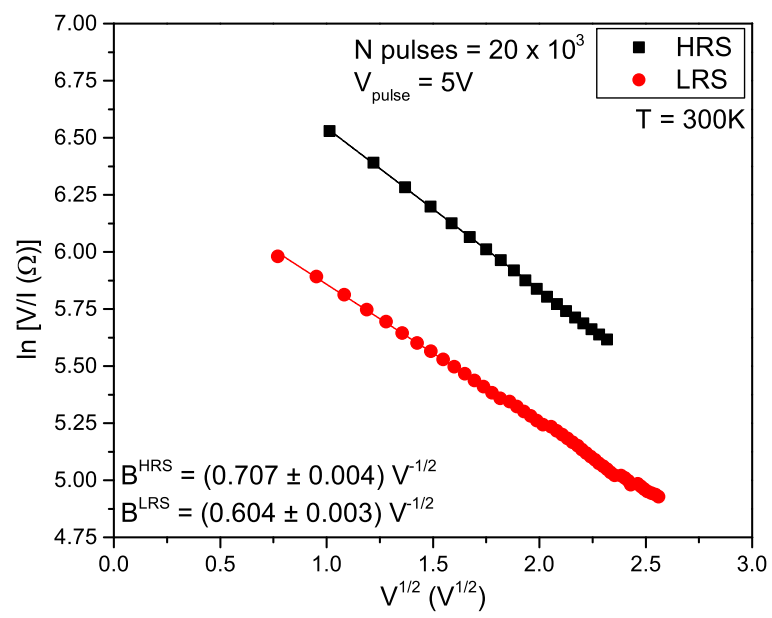

FIG. 3. $\ln (V / I)$ plotted against $V^{1 / 2}$ for the LRS and the HRS, achieved with a set and a reset burst of $N=20 \times 10^{3}$ pulses with $V_{\text {pulse }}= \pm 5 \mathrm{~V}$ at $300 \mathrm{~K}$, respectively. The lines are fits to the P-F emission (Eq. (3)). The fitted $B$ values of each state are indicated. where $R_{0}$ is a pre-factor that will be discussed later, associated with the geometric factor of the conducting path, the electronic drift mobility $(\mu)$, and the density of states in the conduction band. $E_{t}$ is the trap energy level, $k_{B}$ is the Boltzmann constant, $q$ is the electron charge, $\epsilon$ is the dielectric constant of the oxide, and $d$ is the distance between electrodes. The voltage-dependent contact resistance can then be expressed as

$$
R=\frac{V}{I_{P-F}}=R_{0} \exp \left[E_{t} /\left(k_{B} T\right)-B V^{1 / 2}\right] .
$$

From the $B$ values obtained by fitting, the data presented in Fig. 3 with Eq. (3) and by assuming that the dielectric constant of $\mathrm{YBCO}^{23}$ is of the order of $\epsilon \simeq 200 \times 10^{-12} \mathrm{~F} / \mathrm{m}$, the characteristic distance $d$, where most of the voltage drops occurs, can be estimated. We obtain that $d^{H R S} \simeq 0.8 \mu \mathrm{m}$ and $d^{L R S} \simeq 1.1 \mu \mathrm{m}$, which corresponds to a size much lower that the distance between contacts $(\sim 500 \mu \mathrm{m})$, in accordance to the previous results that indicate that the RS-active-region remains limited to a small zone near the interface. ${ }^{24}$

As the $R(T)$ measurements were performed using low currents, the remnant non-linear (NL) contact resistance (Eq. (3)) should be nearly ohmic when $V \ll V_{L}$, where $V_{L}=\pi \epsilon d E_{t}^{2} / q^{3}$. In that case, Eq. (3) can be simplified as

$$
R \simeq R_{0} \exp \left[E_{t} /\left(k_{B} T\right)\right] .
$$

Fig. 4 shows the temperature dependence of the $\mathrm{Au} /$ YBCO contact resistance in both states (LRS and HRS) plotted as $\ln (\mathrm{R})$ vs $T^{-1}$ in the $240 \mathrm{~K}$ to $300 \mathrm{~K}$ temperature range. The $V_{\text {pulse }}$ sensitivity of $R^{H R S}(T)$ can be observed in Fig. 5, where $\ln (\mathrm{R})$ vs $T^{-1}$ is shown for varying $V_{\text {pulse }}$ and a fixed $N=500 \times 10^{3}$. Similar results were obtained for the whole set of $N$ values explored (not shown here). The nearly straight line followed by the data in this temperature range indicates good agreement with Eq. (4), implying that $R_{0}$ and $E_{t}$ should be practically temperature-independent. $E_{t}\left(V_{\text {pulse }}\right)$ and $R_{0}\left(V_{\text {pulse }}\right)$ for $N=500 \times 10^{3}$ pulses can then be extracted for both states (LRS and HRS) by using Eq. (4) to fit the data presented in Figs. 4 and 5. A similar procedure was followed for other values of $N$.

The fitting $E_{t}^{H R S}\left(V_{\text {pulse }}\right)$ values are shown in Fig. 6 for the whole set of $N$ pulses per burst explored. The obtained $E_{t}$ 


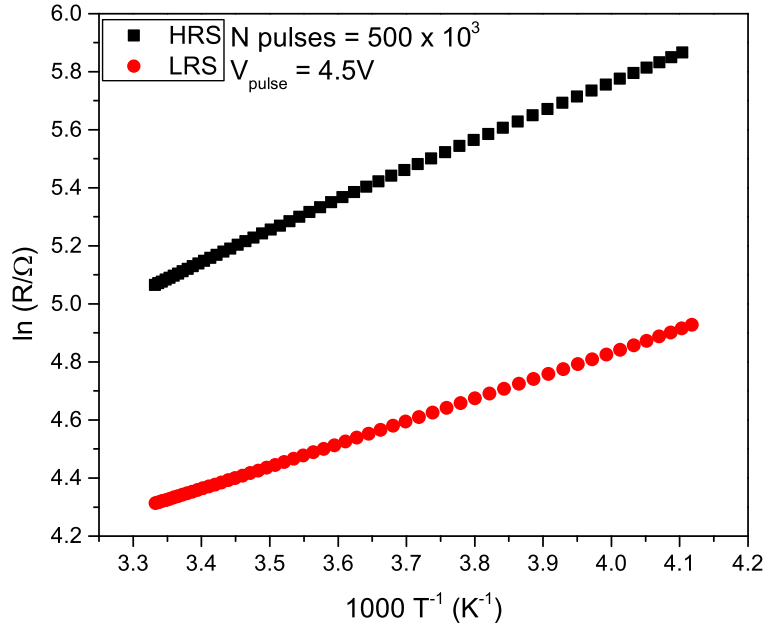

FIG. 4. $\ln (R)$ vs. $T^{-1}$ for the LRS and the HRS, achieved at room temperature with a set and a reset burst of $N=500 \times 10^{3}$ pulses with $V_{\text {pulse }}= \pm 5 \mathrm{~V}$, respectively.

values for both states and for all pulsing treatments are within the $0.06 \mathrm{eV}$ to $0.11 \mathrm{eV}$ range, which validate the approximation made to obtain Eq. (4) $\left(V_{L} \sim 10 \mathrm{~V}\right)$. It can be observed that in the low voltage region $\left[V_{\text {pulse }}<V_{c}(N)\right]$, $E_{t}^{H R S}$ is nearly voltage independent, but decreases with increasing $N$ as a consequence of the decrease of $E_{t}^{L R S}$, due to the set pulsing treatment (see Fig. 1). For $V_{\text {pulse }} \geq$ $V_{c}(N), E_{t}^{H R S}$ increases linearly with $V_{\text {pulse }}$ with a slope of $0.025 \mathrm{eV} \mathrm{V}^{-1}$ and becomes less $N$-dependent. This voltage dependence with a relatively low $\mathrm{N}$-dependence is in accordance with the previously observed behavior, ${ }^{18}$ mentioned as a Basquin-like law. This law indicates that the amplitude of the RS change $(\alpha)$ is more sensible to a voltage variation than to a change in the number of pulses. In this way, to produce the same RS effect, $N$ should be increased by 3 orders of magnitude if $V_{\text {pulse }}$ is reduced to half its value.

The obtained $R_{0}\left(V_{\text {pulse }}\right)$ dependence for different bursts lengths is shown in Fig. 7. Aside from the noisy behavior, two regions can be observed for $E_{f}$ : a voltage independent region for $V_{\text {pulse }}<V_{c}(N)$ and, surprisingly, a tendency to decrease for $V_{\text {pulse }} \geq V_{c}(N)$.

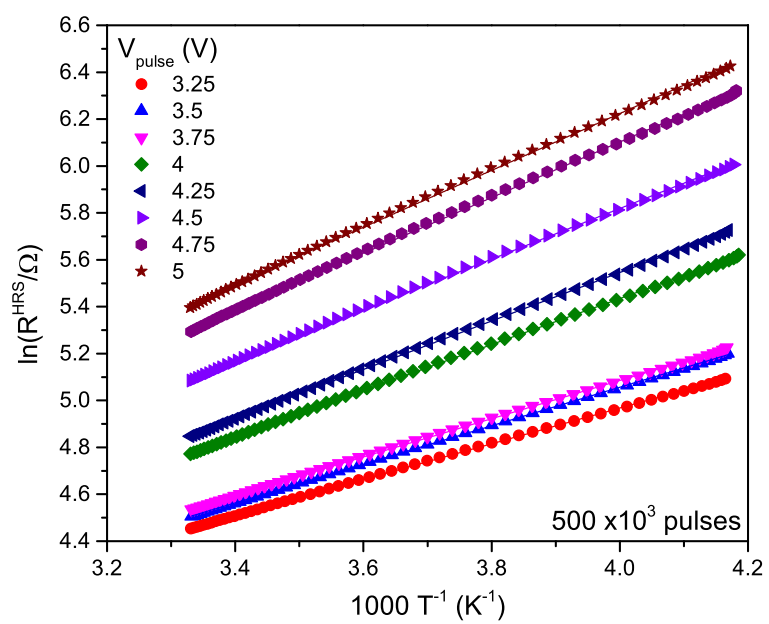

FIG. $5 . \ln (R)$ vs. $T^{-1}$ for the HRS, achieved at $300 \mathrm{~K}$ with a reset burst of $N=500 \times 10^{3}$ pulses with varying pulse amplitudes.

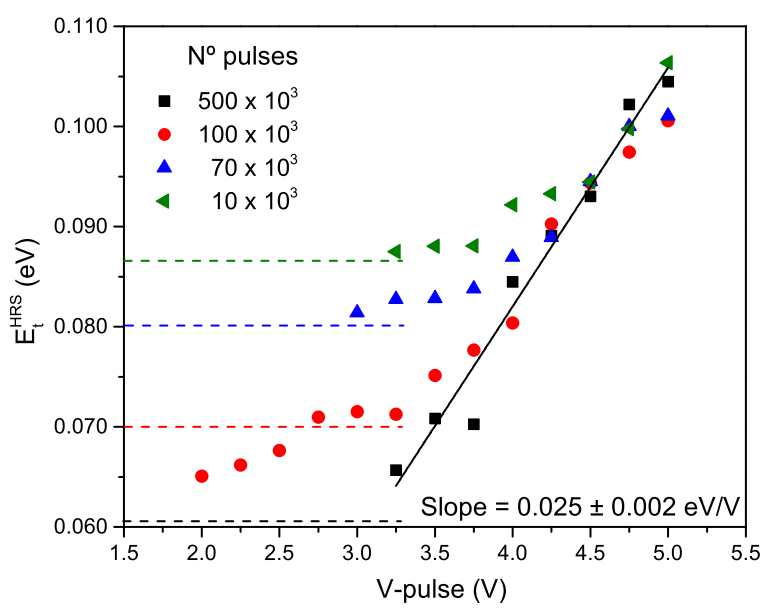

FIG. 6. Trap energy level of the HRS $\left(E_{t}^{H R S}\right)$ as a function of $V_{\text {pulse }}$, for different number $\mathrm{N}$ of applied pulses per burst. The dashed lines correspond to the values of the trap energy level for the LRS $\left(E_{t}^{L R S}\right)$, which are practically $V_{\text {pulse }}$-independent. The solid line indicates a linear fit of the data for $V_{\text {pulse }}>V_{c}(N)$.

On one hand, as our results indicate that the main conduction mechanism through the $\mathrm{Au} / \mathrm{YBCO}$ interface is a $\mathrm{P}-\mathrm{F}$ emission, we have to consider that transport properties are dominated by a carrier-trap region in the YBCO part of the junction. In that sense, YBCO in this interface-active-region cannot be in a metallic-like state, even at room temperature or in the LRS, which indicates that the average density of oxygen-vacancies $(\delta)$ should be roughly in the $0.7-1.0$ range. ${ }^{25}$ For these $\delta$ values, YBCO is not superconducting and has resistivities in a broad range, from $\sim 4$ to $\sim 4000 \mathrm{~m} \Omega$ $\mathrm{cm}^{26,27}$ Oxygen vacancy zones can then be considered as the positively charged traps that will capture electrons, impeding hole mobility. They are, up until now, associated with the microscopic origin of the bipolar RS effect, and models based on their electromigration manage to describe nontrivial experimental results. ${ }^{5}$ Here, the fact that $E_{t}$ increases with increasing $V_{\text {pulse }}$ may indicate that oxygen is not removed uniformly from the YBCO lattice, but in a correlated manner, increasing the size of oxygen-depleted

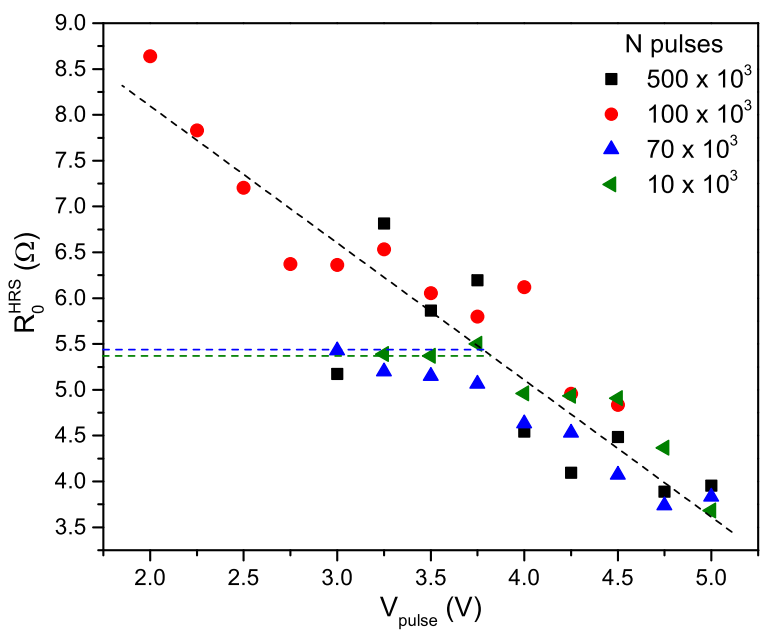

FIG. 7. $R_{0}$ for the HRS as a function of $V_{\text {pulse }}$ for varying burst lengths. The dashed lines are the values of $R_{0}$ for the LRS, which are nearly $V_{\text {pulse }}-$ independent. 
spots, probably because the local electric field is larger in these zones. As will be discussed later, the decrease in $R_{0}$ with $V_{\text {pulse }}$ can be related to an increase in the cross-sectional area of a low-conducting filament in the HRS.

On the other hand, it was shown ${ }^{26,27}$ that the electrical conduction of low-oxygen-content YBCO is by a 2D-VRH mechanism, as oxygen vacancies introduce disorder in the electronic potential. ${ }^{28}$ At the same time, we may consider the scenario depicted here, where part or the totality of the hopping carriers can be trapped in deeper potential wells, associated with the electric-pulse-induced oxygen vacancy spots. In this case, their number will be limited, introducing a characteristic de-trapping energy $\left(E_{t}\right)$ and the NL characteristics of the P-F effect.

The standard 2D-VRH resistivity $\left(\rho^{V R H}\right)$ of the oxygendepleted YBCO near the interface can be expressed as

$$
\rho^{V R H}=\rho_{0} \exp \left[\left(T_{0} / T\right)^{1 / 3}\right]
$$

where $T_{0}$ is a parameter related to the localization length and to the density of states at the Fermi energy, and

$$
\rho_{0}=\left(k_{B} T\right) /\left(n q^{2} \nu_{p h} R_{h}^{2}\right),
$$

where $n$ is the density of carriers, $q$ is the electron's charge, $\nu_{p h}$ is a characteristic phonon-assisted hopping frequency, and $R_{h}$ is the hopping distance.

This expression should be modified to consider the influence of traps. If the density of traps $n_{t} \geq n$ and if we assume that at $\mathrm{T}=0 \mathrm{~K}$ all the electrical carriers are trapped, only those de-trapped or ionized $\left(n_{i}\right)$ will participate in the VRH conduction. As the barrier to overcome, $\left(E_{t}\right)$ can be reduced by the $\mathrm{P}-\mathrm{F}$ effect, then $n_{i}=n \exp \left(-E_{t} / k_{B} T+B V^{1 / 2}\right)$. Within this scenario, the contact resistance associated with an oxygen-depleted YBCO zone of characteristic length $d$ and a conducting area $S$ can be rewritten as

$$
\begin{aligned}
R & =\frac{d}{S} \rho^{V R H} \exp \left[E_{t} /\left(k_{B} T\right)-B V^{1 / 2}\right] \\
& =\frac{d}{S} \rho_{0} \exp \left[\left(T_{0} / T\right)^{1 / 3}+E_{t} /\left(k_{B} T\right)-B V^{1 / 2}\right]
\end{aligned}
$$

In fact, as the disorder that produces the VRH conduction can be associated with an oxygen-vacancy random distribution, which may produce trapping centers for the carriers that yield to a P-F emission, both conduction mechanisms may coexist or dominate, depending on different factors as stated in Eq. (7), VRH should be the main mechanism for temperatures $T>T_{L}$ with $T_{L}=\left(\frac{\left(E_{t} / k_{B}\right)^{3}}{T_{0}}\right)^{1 / 2}$. While for $T<T_{L}$, the conduction should be of the P-F type. In the same way, NL effects should be observed for voltages $V \gtrsim V_{L}$ in the P-F case and may even be detected when $V \gtrsim 0.1 V_{L}$. While in the VRH regime, they should be noticed for a low temperature region, where $T \lesssim \frac{q^{9 / 4} V^{3 / 4}}{k_{B}^{3 / 2}(\pi \epsilon d)^{3 / 4} T_{0}^{1 / 2}}$. In other words, depending on the degree of disorder, on the trap energy level and on the temperature range explored, lowoxygen-content YBCO can present, intrinsically, a VRH or a $\mathrm{P}-\mathrm{F}$ regime, as well as NL-IV characteristics. The VRH + the
NL-IV regime was in fact observed previously ${ }^{26}$ for $\mathrm{YBa}_{2} \mathrm{Cu}_{3} \mathrm{O}_{6}$, where the electrical transport properties, measured in a four terminal configuration at $40 \mathrm{~K}$ to $300 \mathrm{~K}$, were well described by a 2D-VRH conduction and showed the presence of a NL regime at low temperatures, whose origin has not been discussed so far. Conversely, our results in the $\mathrm{Au} / \mathrm{YBCO}$ junctions for the $240 \mathrm{~K}$ to $300 \mathrm{~K}$ temperature span indicate that the P-F emission is favored over the VRH regime. In this way, the interfacial region of YBCO may present higher trap energy levels (oxygen-depleted spots) and a less disordered crystal structure than that existing in the oxygen-depleted YBCO with $\delta \sim 1$. As mentioned, this can be related to a correlated oxygen vacancy distribution, which increases the trap energy level without increasing the overall disorder, as in a random vacancy distribution. A sketch of these possible two scenarios is presented in Fig. 8(a).

The mentioned low conductivity of the pristine $\mathrm{YBCO}$ in contact with the Au interface may be a consequence of the higher oxidation energy of $\mathrm{Au}$ when compared to YBCO. One possibility (see Fig. 8(b)) is that Au depleted oxygens from the interfacial $\mathrm{YBCO}$, generating a disordered potential with traps for the charge carriers.

Just to give an order of magnitude, we can assume that this interfacial YBCO has a mean density of vacancies of $\delta \simeq 0.7$ to 1 per unit cell volume $\left(n_{t} \sim 6 \delta \times 10^{21} \mathrm{~cm}^{-3}\right)$ in the LRS and in the HRS, respectively. In this case, $\rho^{V R H}$ should range from $4 \mathrm{~m} \Omega \mathrm{cm}$ to $4000 \mathrm{~m} \Omega \mathrm{cm}^{25,26}$ In these
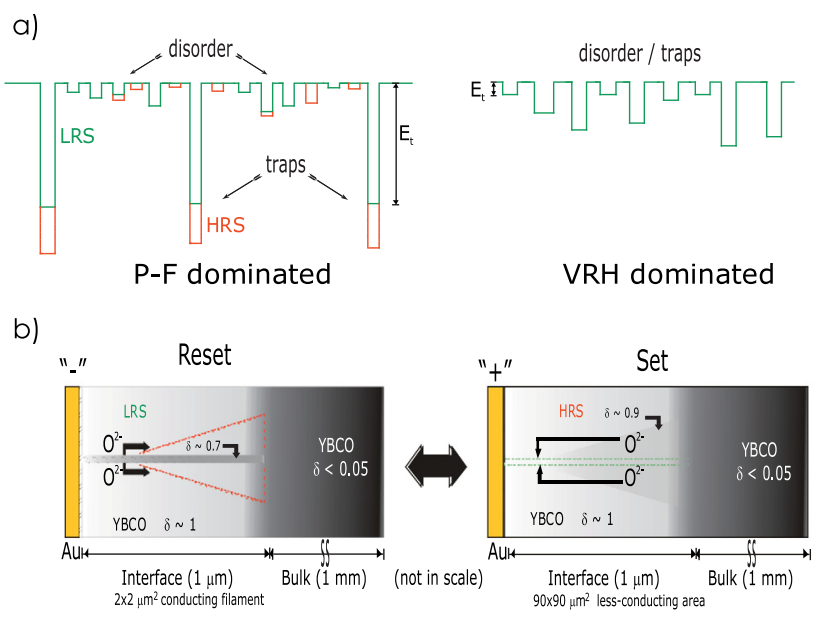

FIG. 8. (a) Schematic representation of the spatial distribution of the disordered electronic potential background with deep traps (P-F dominated transport) and with shallow traps (VRH dominated transport). In the former case, increasing the amplitude of electric pulses produces deeper traps, clearly differentiated from the background, while in the latter case, traps and background disorder are indistinguishable. (b) Sketch of the Au/YBCO interface. The P-F IV characteristics indicate that the interfacial YBCO corresponds to a $\sim 1000 \times 1000 \times 1 \mu \mathrm{m}^{3}$ volume with a random distribution of vacancies, although spots of many surrounding vacancies acting as carrier-traps should be formed. After the set pulsing treatment, a $\sim 2 \times 2 \times 1 \mu \mathrm{m}^{3}$ conducting filament is formed in the LRS by electromigration of oxygens from the bulk, in accordance to the previous models. ${ }^{5}$ The oxygen content of this filament can be increased up to the optimum oxygen content, creating a superconducting filament of reduced geometric factor. ${ }^{8}$ Oxygen from this filament is then removed when applying the reset pulses, generating the HRS, which corresponds to a less conducting and thicker filament $\left(\sim 90 \times 90 \times 1 \mu \mathrm{m}^{3}\right)$, probably of conical shape. ${ }^{29}$ 
conditions, the conduction area $S$ can be roughly estimated by considering Eq. (7) and the low voltage measurements of the contact resistance at $300 \mathrm{~K}$. The obtained values, $S^{L R S} \sim$ $2 \times 2 \mu \mathrm{m}^{2}$ and $S^{H R S} \sim 90 \times 90 \mu \mathrm{m}^{2}$, indicate the filamentary nature of the area modified by the RS pulsing treatments. The dispersive diffusion of oxygen may be the reason for the increase in $S^{H R S}$ when compared to $S^{L R S}$. Although $\rho^{V R H}$ for YBCO increases with increasing $\delta$, the increase in $S^{H R S}$ determines the decrease in $R_{0}$ with $V_{\text {pulse }}$ (see Fig. 7). Local measurements of the oxygen content near the interface are needed to prove the validity of this sketched scenario.

\section{CONCLUSIONS}

In summary, transport measurements performed on $\mathrm{Au} /$ optimally doped YBCO as a function of temperature and electric field showed an electrical conduction dominated by a P-F emission mechanism. As this is a bulk property of the interfacial YBCO, we inferred that in a $1 \mu \mathrm{m}$ region near the interface, YBCO is oxygen-depleted, in a way that favors the existence of traps for the carriers. The energy level of these traps $\left(E_{t}\right)$ can be increased linearly by increasing the amplitude of the voltage pulses. This result can be interpreted as an indication that the electromigration of oxygens may not be produced randomly but in a correlated manner. The number of applied pulses $N$ produces less changes on $E_{t}$ than those obtained by increasing $V_{\text {pulse }}$, in accordance with a previously stated similarity with the stress-lifetime Basquin law. We also proposed a simple description of the intrinsic transport scenario for oxygen-depleted YBCO, where the VRH carriers may be trapped by oxygen vacancies, yielding to P-F emission or to VRH conduction, with or without NL effects, depending on the temperature range explored. Finally, an estimation of the conduction area for both states reveals the filamentary nature of the zone influenced by the pulsing treatments.

\section{ACKNOWLEDGMENTS}

We would like to acknowledge financial support by CONICET Grant Nos. PIP 112-200801-00930, PICT 20130788, and UBACyT 20020130100036BA (2014-2017). We also acknowledge V. Bekeris and F. Acha for a critical reading of this paper and D. Giménez, E. Pérez Wodtke and D. Rodríguez Melgarejo for their technical assistance.

${ }^{1}$ G. W. Burr, B. N. Kurdi, J. C. Scott, C. H. Lam, K. Gopalakrishnan, and R. S. Shenoy, IBM J. Res. Dev. 52, 449 (2008).

${ }^{2}$ J. J. Yang, D. B. Strukov, and D. R. Stewart, Nat. Nanotechnol. 8, 13 (2013).

${ }^{3}$ A. Sawa, Mater. Today 11, 28 (2008).

${ }^{4}$ R. Waser and M. Aono, Nat. Mater. 6, 833 (2007).

${ }^{5}$ M. J. Rozenberg, M. J. Sánchez, R. Weht, C. Acha, F. Gomez-Marlasca, and P. Levy, Phys. Rev. B 81, 115101 (2010).

${ }^{6}$ S. M. Sze and K. K. Ng, Physics of Semiconductor Devices (John Wiley \& Sons, 2006).

${ }^{7}$ F-Ch. Chiu, Adv. Mater. Sci. Eng. 2014, 578168 (2014).

${ }^{8}$ C. Acha and M. J. Rozenberg, J. Phys.: Condens. Matter 21, 045702 (2009). ${ }^{9}$ C. Acha, Physica B 404, 2746 (2009).

${ }^{10}$ A. Plecenik, M. Tomasek, T. Plecenik, M. Truchly, J. Noskovic, M. Zahoran, T. Rocha, M. Belogolovskii, M. Spankova, S. Chromik, and P. Kus, Appl. Surf. Sci. 256, 5684 (2010).

${ }^{11}$ C. Acha, J. Phys. D: Appl. Phys. 44, 345301 (2011).

${ }^{12}$ A. Schulman and C. Acha, MRS Proc. 1337, mrss11-1337-q10-07 (2011).

${ }^{13}$ T. Plecenik, M. Tomasek, M. Belogolovskii, M. Truchl, M. Gregor, J. Noskovic, M. Zahoran, T. Roch, I. Boylo, M. Spankova, S. Chromik, P. Kus, and A. Plecenik, J. Appl. Phys. 111, 056106 (2012).

${ }^{14}$ A. Schulman, M. J. Rozenberg, and C. Acha, Phys. Rev. B 86, 104426 (2012).

${ }^{15}$ L. Porcar, D. Bourgault, J. M. Barbut, M. Barrault, P. Germi, and R. Tournier, Physica C 275, 293-298 (1997).

${ }^{16}$ H. Takagi, S. Uchida, H. Iwabuchi, H. Eisaki, K. Kishio, K. Kitazawa, K. Fueki, and S. Tanaka, Physica B+C 148, 349 (1987).

${ }^{17}$ R. J. Cava, A. W. Hewat, E. A. Hewat, B. Batlogg, M. Marezio, K. M. Rabe, J. J. Krajewski, W. F. Peck, Jr., and L. W. Rupp, Jr., Physica C 165, 419 (1990).

${ }^{18}$ A. Schulman and C. Acha, J. Appl. Phys. 114, 243706 (2013).

${ }^{19}$ N. Ghenzi, M. J. Sánchez, M. J. Rozenberg, P. Stoliar, F. G. Marlasca, D. Rubi, and P. Levy, J. Appl. Phys. 111, 084512 (2012).

${ }^{20}$ W. Schutz, Eng. Fract. Mech. 54, 263 (1996).

${ }^{21}$ S. Suresh, Fatigue of Materials (Cambridge University Press, 1998).

${ }^{22}$ J. G. Simmons, Phys. Rev. 155, 657 (1967).

${ }^{23}$ J. Mannhart, Supercond. Sci. Technol. 9, 49 (1996).

${ }^{24}$ Z.-H. Wang, Y. Yang, L. Gu, H.-U. Habermeier, R.-C. Yu, T.-Y. Zhao, J.R. Sun, and B.-G. Shen, Nanotechnology 23, 265202 (2012).

${ }^{25}$ B. Wuyts, V. V. Moshchalkov, and Y. Bruynseraede, Phys. Rev. B 53, 9418 (1996).

${ }^{26}$ A. Matsushita, T. Oguchi, K. Kimura, T. Matsumoto, T. Hatano, K. Ogawa, and S. Takayanagi, Jpn. J. Appl. Phys., Part 2 26, L1953 (1987).

${ }^{27}$ F. P. Milliken, T. Doderer, R. H. Koch, and C. C. Tsuei, Phys. Rev. B 62, $9143(2000)$

${ }^{28}$ B. I. Shklovskii and A. L. Efros, Electronic Properties of Doped Semiconductors (Springer-Verlag, 1984).

${ }^{29}$ K. M. Kim and Ch. S. Hwang, Appl. Phys. Lett. 94, 122109 (2009). 